\title{
Micro-Domain Force Estimation Using Hall-Effect Sensors for a Magnetic Microrobotic Station*
}

\author{
Moein MEHRTASH ${ }^{* *}$ and Mir Behrad KHAMESEE** \\ ** Mechanical and Mechatronics Engineering Dept., University of Waterloo \\ 200 University Ave., Waterloo, Ontario N2L-3G1, Canada \\ E-mail:khamesee@uwaterloo.ca
}

\begin{abstract}
This paper introduces a novel micro-domain force estimation method for applications in a magnetic-haptic micromanipulation platform (MHMP). The MHMP employs the magnetic levitation technology in micro-domain worlds for ultra-high precision micromanipulation. In the MHMP, a microrobot that consists of a magnetic head and a body that includes electronic parts and an end-effector is manipulated by regulating an external magnetic field. The MHMP has been equipped with a haptic technology to allow a human operator to feel micro-domain environments and to intervene in dexterous tasks due to the poor knowledge from micro-worlds. To preserve a high feeling of a micro-domain environment for a human operator, the applied force/torque from the environment to the microrobot are required to be directly measured by specific sensors. Due to the size restriction, attaching force sensors to our microrobot is impractical. Therefore, we use a combination of Hall-effect sensor in the structure of the MHMP to estimate a single-axis force, eliminating the need for sensors on the microrobot. The Hall-sensors measure the magnetic flux and determine the location of the horizontally zero magnetic field gradient, $B_{\max }$ location. It was realized that the applied force from the environment to the microrobot is linearly proportional to the distance of the microrobot from the $B_{\max }$ location. The magnetic force which is equal to the environment force is calibrated using a cantilever deflection. The developed micro-domain force estimation method is verified experimentally, and it was demonstrated that this method has promising potential in estimating the environmental force applied to the microrobot in a non-contact way.
\end{abstract}

Key words : Magnetic Levitation, Micro-Domain Force Measurement, Hall-Effect Sensor, Micromanipulation

\section{Introduction}

In recent years, there has been an increasing interest in advancement of microrobotic systems in biological and biomedical applications. Such systems are employed for cell/scaffold micromanipulations $^{(1)}$, cell injections ${ }^{(2)}$, microsurgery ${ }^{(3)}$, and micro-drug delivery ${ }^{(4)}$. Untethered microrobotic systems are one of the most widely developing groups of microrobotic systems that have been extensively explored for biological and biomedical micromanipulations in recent decades. These untethered systems show promising approach in accommodating the on-board power supply limitations, the power required for the microrobot locomotion is transferred to the microrobot from a macro-domain power source.

A magnetic-haptic micromanipulation platform (MHMP) ${ }^{(5)}$ has been developed at the Maglev Microrobotics Laboratory, University of Waterloo for biological and biomedical micromanipulations, schematically shown in Fig. 1. The MHMP consists of three basic subsystems as follows: a magnetic untethered microrobot system (MUMS), a haptic device, and a bilateral teleoperation system. The MUMS uses the magnetic-based propulsion technol-

${ }^{*}$ Received 24 June, 2012 (No. 12-0273) [DOI: $10.1299 /$ jamdsm.7.2]

Copyright (c) 2013 by JSME 
MHMP Site

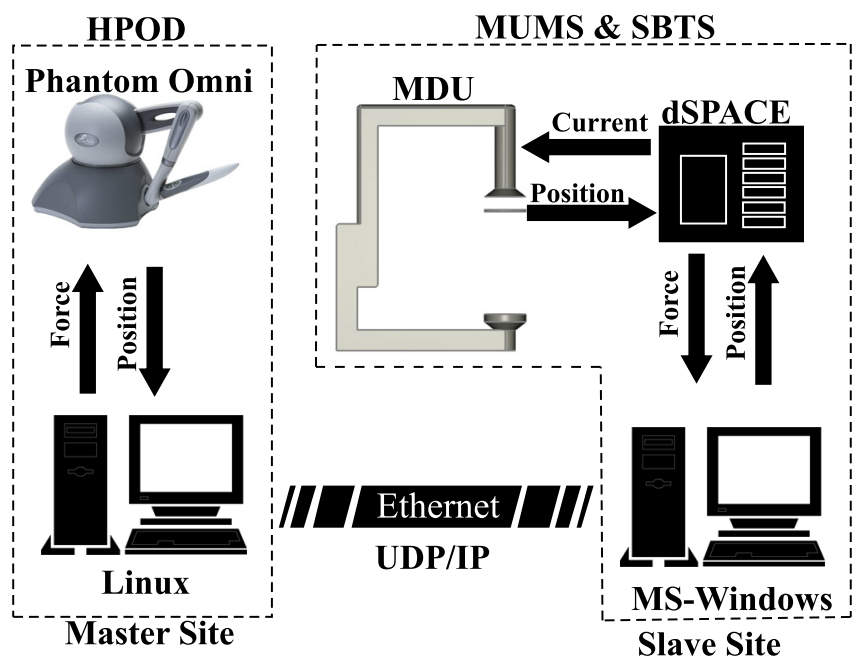

Fig. 1 Schematic representation of the magnetic haptic manipulation platform $(\text { MHMP) })^{(5)}$

ogy that produces a bio-compatible environment. The MHMP utilizes a commercial haptic interface as its master side that operates by a human operator. A bilateral macro-micro teleoperation technique has been employed for the telepresence of the human operator in the task's environment. During our previous studies, we developed the MHMP by which the human operator can feel the hard contact (high stiffness environment) during tasks if the microrobot confront a stiff environment. In the MHMP development process, we have equipped this platform to transfer the soft contact (small stiffness environment) feeling to the human operator. Thus, the applied force from the environment to microrobot is needed to be measured and then transfered to the human operator's hand.

Many methods for measuring force from the micro-Newton to milli-Newton range have been developed, such as atomic force microscopes ${ }^{(6)}$, microscales, piezoresistive cantilevers (7)-(12) and capacitive force sensors ${ }^{(13)}$. These force measurement systems have been employed in the research field of microsystem and microassembely, as well as biomedical/ biological research. However, attaching such measurement systems to the MHMP's microrobot is impracticable due to: 1) micro-scaled sensors is needed to be integrated with the microrobot for measuring forces; thus, the overall size and weight of the microrobot is largely increased, 2) to maintain free levitation, the microrobot needs to be equipped with an onboard wireless communication device to transfer the sensors' measurements, 3) the microsized force sensors are expensive and very sensitive to the physical parameters of an environment. This study proposes a novel micro-domain force estimation method eliminating the need of mounting a force sensor to the microrobot. The non-contact force estimation is carried out based on the magnetic flux measurements.

This paper is organized as follows. Section I has already introduced the need for measuring the micro-domain force for a microrobotic platform. Section II explains the principal of magnetic propulsion used in the development of the MHMP. Section III describes the contribution of the proposed force measurement systems for the MHMP. Section IV explains the rule of Hall-effect sensors in force estimation. Finally, Section V discusses the calibration of the force estimation method and experimental verifications.

\section{Background}

This section, firstly, demonstrates the overall structure of the Magnetic-Haptic Micromanipulation platform (MHMP) and describes the functionalities of its components. Then, the concept of non-contact manipulation using magnetic propulsion are reviewed and mathemat- 
ically represented.

\subsection{Description of Magnetic-Haptic Micromanipulation platform (MHMP)}

The magnetic-haptic micromanipulation platform (MHMP) has been developed at Maglev Microrobotics Laboratory, University of Waterloo, and introduced in Ref. (5) with promising potential for extensive micromanipulation applications. This platform includes two sites- a master and a slave- that can communicate with each other by analog and/or Ethernet connections. The Ethernet/UDP communication channel provides fast data transferring for this real-time system, and enables the human operator in the master site to conduct remotely a task in the slave site connected to the Internet; Fig. 1 presents a schematic diagram of the MHMP.

The slave site includes the magnetic microrobotic station (MMS) and a scaled bilateral teleoperation system (SBTS). The MMS is a magnetic untethered microrobotic system (MUMS) that consists of two main separated components: a magnetic drive unit (MDU) and a microrobot. The MDU produces and regulates the magnetic field for propelling the microrobot in a non-contact way; Section 2.2 reviews the principle of magnetic propulsion and the magnetic force model produced by the MDU. The MDU has been equipped with three high precision laser micrometers to determine the position of the microrobot in three axes; the laser sensors provide the accuracy of 2-8 $\mu \mathrm{m}$ [the highest accuracy at the center region of micrometer's working space and the lowest at the boundaries]. The MDU uses three PID controllers to stabilize the microrobot's motion inside a cubic workspace. The controllers for horizontal axes provide $1.5 \mu \mathrm{m}$ as the root-mean-square, RMS, of the position error. A 16-bit analogue card is used to capture the laser reading in the working range of \pm 5 volt that causes $0.45 \mu \mathrm{m}$ measurement error. Considering the hardware errors, the accuracy of positioning the microrobot can be estimated as 4-12 $\mu \mathrm{m}$ in the working envelope of the MHMP as $20 \times 20 \times 30$ $\mathrm{mm}^{3(5)}$. The MHMP's microrobot is made of two main components: a magnetic head, a body that carries electronic parts, and an end-effector.

The bilateral macro-micro teleoperation technique is employed in the STBS for the telepresence of a human operator in the micro-domain world. The SBTS implements macromicro scaling for both position and force to make a micro-domain task more comfortable for the human operator. The gain-switching control scheme ${ }^{(14)}$ is utilized as a strategy of the SBTS for the MHMP to improve the transparency of position-error-based teleoperation for two cases: 1) free motion, and 2) bump into a hard contact. However, the transparency and fidelity of the SBTS can be improved if the SBTS's controller is fed with the measurements slave-side force sensors, 4-channel bilateral teleoperation ${ }^{(15)}$.

The master site includes the haptic station. This station consists of a haptic Phantom omni device (HPOD) and a server computer. The human operator can control the microrobot's position by moving the HPOD's stylus. In addition, the HPOD provides force-feedback to the human operator's hand. A Linux server computer is employed to communicate with the slave site. This server sends the position command from master site to slave site and also receives the calculated force-feedback from the slave site through analog and/or Ethernet/UDP. More details of the MHMP can be reviewed in Ref. (5).

\subsection{Principle of Magnetic Propulsion and Modeling}

The magnetic propulsion originates from the interaction of a magnetic material with the external magnetic field. Placing the permanent magnet with an inherent magnetization $\mathbf{M}$ in an external magnetic field $\mathbf{B}$ raises the total potential energy and proceeds a force exerted on the unit volume of a permanent magnet, as in Ref. (5),

$$
f=+\nabla(\mathbf{M} \cdot \mathbf{B})
$$

This external magnetic field also applies magnetic toque $\tau=\mathbf{M} \times \mathbf{B}$ to the unit volume of the permanent magnet. This magnetic torque tends to orient the permanent magnet parallel 


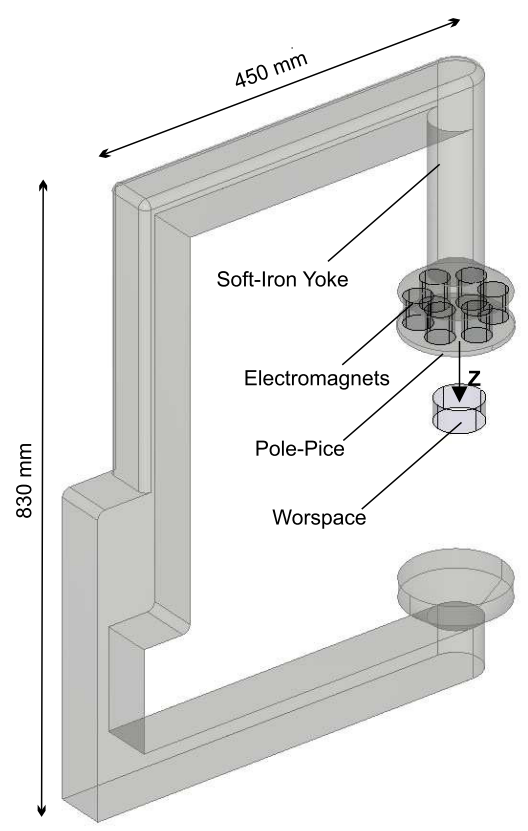

(a) Schematic view of the MDU

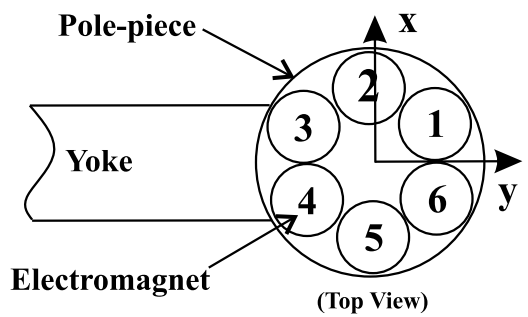

(b) Arrangement of electromagnets

Fig. 2 Basic components of the magnetic drive unit (MDU)

to the external magnetic field's vector. Therefore, the forces and torques experienced by the permanent magnet can be controlled by regulating the external magnetic field. More details of controlling the magnetic forces and torques have been reviewed in Ref. (5), (16), (17).

The magnetic untethered microrobotic system (MUMS), as the heart of micromanipulation platform, is made up of two separated subsystems: a magnetic drive unit (MDU) and a microrobot. The drive unit is advanced to generate the magnetic field for propelling the microrobot in an enclosed environment, shown in Fig. 2 schematically. The drive unit consists of six-pair of electromagnets, a disc pole-piece for connecting the magnetic poles, and a yoke. The effectiveness of this structure for the 3D position controlling of the microrobots was reported in ${ }^{(5),(16),(17)}$. The maximum magnetic field in z-direction produced by the MDU is in the range of 0.1-0.2 Tesla inside the workspace. The resolution of the generated magnetic field by MDU is $0.19 \mu \mathrm{T}$.

In this study, we model the microrobot's head with magnetization $\mathbf{M}$ as a single dipole permanent magnet, and it is assumed that $\mathbf{M}=\left[\begin{array}{lll}0 & 0 & M\end{array}\right]$. Furthermore, For a small permanent magnet (PM), the magnetization can be taken as a constant and as uniformly distributed throughout the volume. Therefore, the induced magnetic force due to the external magnetic field can be represented by

$$
f=M\left(\nabla B_{z}\right)
$$

This mathematical relation demonstrates the changes of magnetic force with the gradients of the z-component of the magnetic field. In theory, the permanent magnet placed in an external magnetic field is inclined to move toward the minimum total potential energy location; i.e. $B_{\max }$ point - $B_{\max }$ point signifies the location of the maximum magnetic field on the horizontal plane which the PM is hanged on. If the PM is in a steady state condition, its weight is balanced by the vertical magnetic force, and the horizontal forces equal zero, since no horizontal force occurs in the $B_{\max }$ position. Hence, the PM can be manipulated by regulating the gradients of $B$ and controlling the $B_{\max }$ location. Figure 3 demonstrates the changes of $B_{\max }$ locations by the variations of the coils' currents of the electromagnets. This figure also represents the capability of the MDU in producing a unique $B_{\max }$ location in the horizontal plane. Based on experimental measurements and numerical analyses, we developed the 

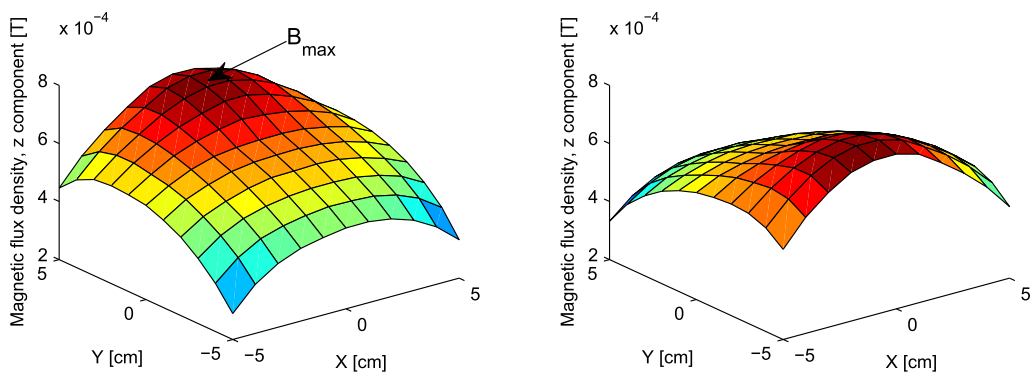

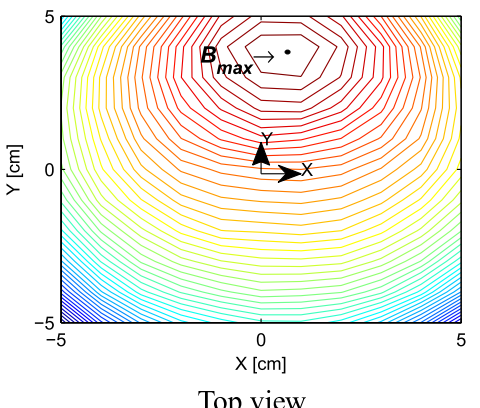

(a)

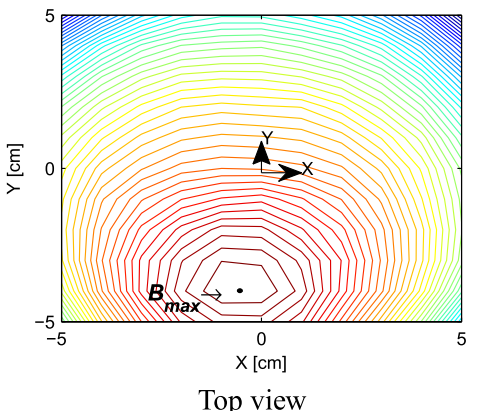

(b)

Fig. 3 Surface and contour plot of magnetic flux density z-component obtained by experimental measurement, (a) and (b) demonstrate the $B_{z}$ in the horizontal plane with the coils' currents with two various patterns $P_{1}$ and $P_{2}$, respectively. The contour plot of the $B_{\max }$ location place under surface plot with the coils' currents of two various patterns $P_{1}$ and $P_{2}$, respectively.

MDU's magnetic force model ${ }^{(5),(17)}$ as

$$
\begin{aligned}
& f_{x}=M \frac{\partial B_{z}}{\partial x}=x / 4\left(\alpha_{x}+3 \alpha_{y}\right)\left(I_{1}+I_{3}+I_{4}+I_{6}\right)+x \alpha_{x}\left(I_{2}+I_{5}\right)+\sqrt{3} / 4 y\left(\alpha_{x}-\alpha_{y}\right) \\
& \left(I_{1}-I_{3}+I_{4}-I_{6}\right)-\sqrt{2} / 2 \beta_{y}\left(I_{1}-I_{3}+I_{4}-I_{6}\right) \\
& f_{y}=M \frac{\partial B_{z}}{\partial y}=y / 4\left(3 \alpha_{x}+\alpha_{y}\right)\left(I_{1}+I_{3}+I_{4}+I_{6}\right)+y \alpha_{y}\left(I_{2}+I_{5}\right)-\sqrt{3} / 4 x\left(\alpha_{x}-\alpha_{y}\right) \\
& \left(I_{1}-I_{3}+I_{4}-I_{6}\right)-1 / 2 \beta_{y}\left(I_{1}+2 I_{2}+I_{3}-I_{4}-2 I_{5}-I_{6}\right) \\
& f_{z}=M \frac{\partial B_{z}}{\partial z}=\left(\alpha_{z} z+\beta_{z}\right)\left(I_{1}+I_{2}+I_{3}+I_{4}+I_{5}+I_{6}\right)
\end{aligned}
$$

where $\alpha_{x}, \alpha_{y}, \alpha_{z}, \beta_{y}$, and $\beta_{z}$ and are magnetic field parameters, determined by experimental measurement and the linear least square curve fitting. The parameter $I_{i}$ presents electromagnet number i's coil current, the electromagnets' arrangements is shown in Fig. 2.

\section{The Concept of Environmental Force Estimation}

The previous section discussed the principle of microrobot's free levitation and the mathematical force model experienced by the microrobot inside the magnetic field produced by the MDU. In a condition that the microrobot is in contact with an environment that applies force $\left(f_{e}\right)$ to the microrobot, the microrobot is not stabilized at the $B_{\max }$ location. The magnetic field produced by the MDU exerts force to the microrobot to move it toward the $B_{\text {max }}$ location, meanwhile the environment applies equal and opposite direction force $\left(f_{e}\right)$ to the microrobot. Thus, the microrobot becomes stable at a location that the magnetic field gradient is not equal to zero in the horizontal motion plane, shown schematically in Fig. 4 [This study focuses on the investigation of environmental force measurement in y-direction; however, the motion study in $\mathrm{x}$ - and z-direction can be performed in the similar way, the reference axis is shown in Fig. 2]. In the steady state condition, the magnetic force applies to the microrobot equals to the environmental force that can be estimated by using Eq. 5 as

$$
f_{y}=f_{e}=y_{r} / 4\left(3 \alpha_{x}+\alpha_{y}\right)\left(I_{1}+I_{3}+I_{4}+I_{6}\right)+y_{r} \alpha_{y}\left(I_{2}+I_{5}\right)-\sqrt{3} / 4 x_{r}\left(\alpha_{x}-\alpha_{y}\right)
$$




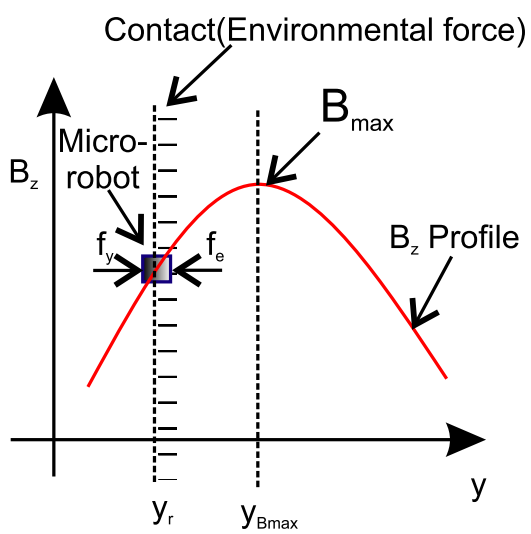

Fig. 4 The schematic representation of the force estimation concept

$$
\left(I_{1}-I_{3}+I_{4}-I_{6}\right)-1 / 2 \beta_{y}\left(I_{1}+2 I_{2}+I_{3}-I_{4}-2 I_{5}-I_{6}\right)
$$

where $x_{r}$ and $y_{r}$ determine the position of the microrobot in the horizontal plane relative to the reference coordinate defined in Fig. 2. Since, it is a single-axis force estimation, y-direction, we assumed the $x_{r}=0$ and keep the vertical position of the microrobot $z_{r}$ equals to $z_{0}$. The microrobot real position can be represents as $y_{r}=y_{B m a x}+\Delta y$, Fig. 4. This relation can then be replaced in Eq. 6 as

$$
\begin{aligned}
f_{y}= & f_{e}=\left(y_{\text {Bmax }}+\Delta y\right) / 4\left(3 \alpha_{x}+\alpha_{y}\right)\left(I_{1}+I_{3}+I_{4}+I_{6}\right)+\left(y_{B \max }+\Delta y\right) \alpha_{y}\left(I_{2}+I_{5}\right) \\
& -1 / 2 \beta_{y}\left(I_{1}+2 I_{2}+I_{3}-I_{4}-2 I_{5}-I_{6}\right)
\end{aligned}
$$

re-arranging this equation as

$$
\begin{aligned}
f_{y}= & f_{e}=\overbrace{\left[\Delta y / 4\left(3 \alpha_{x}+\alpha_{y}\right)\left(I_{1}+I_{3}+I_{4}+I_{6}\right)+\Delta y \alpha_{y}\left(I_{2}+I_{5}\right)\right]}^{A_{1} \neq 0}+ \\
& {\left[y_{B \max } / 4\left(3 \alpha_{x}+\alpha_{y}\right)\left(I_{1}+I_{3}+I_{4}+I_{6}\right)+y_{B \max } \alpha_{y}\left(I_{2}+I_{5}\right)-\right.} \\
& \left.1 / 2 \beta_{y}\left(I_{1}+2 I_{2}+I_{3}-I_{4}-2 I_{5}-I_{6}\right)\right]
\end{aligned}
$$

the term $A_{1}$ is the non-zero part of this force equation, since the magnetic force at the $B_{\max }$ location is zero. Therefore, the force equation can then be derived as

$$
f_{y}=f_{e}=\Delta y / 4\left(3 \alpha_{x}+\alpha_{y}\right)\left(I_{1}+I_{3}+I_{4}+I_{6}\right)+\Delta y \alpha_{y}\left(I_{2}+I_{5}\right)
$$

We assume that the environmental forces apply to the microrobot in y-direction and keep $x_{r}=0, z_{r}=z_{0}$ [environmental force in the $\mathrm{x}$-direction is zero, $f_{x}=0$, and the microrobot's weight is the only force in the z-direction]. Therefore, using Eq. 3 and 5 results in

$$
\begin{aligned}
& I_{1}+I_{2}+I_{3}+I_{4}+I_{5}+I_{6}=C_{1} \\
& I_{1}+I_{4}-I_{3}-I_{6}=0 \Longrightarrow I_{1}+I_{4}=I_{3}+I_{6}=C_{2}
\end{aligned}
$$

where $C_{1}$ and $C_{2}$ are constants for motions in the y-direction; replacing these two relation in Eq. 9 re-presents the force model as

$$
f_{y}=f_{e}=\Delta y \underbrace{\left[\frac{3}{2} \alpha_{x} C_{2}+\frac{1}{2} \alpha_{y}\left(2 C_{1}-3 C_{2}\right)\right]}_{C}=C \Delta y
$$

This equation demonstrates that the magnetic force applies to the microrobot is linearly proportional to the distance of the microrobot from the $B_{\max }$ location. Thus, by determining the $B_{\max }$ location and the constant $C$ in Eq. 12, the environmental force in the steady-state condition can be estimated. The next section reviews a method to obtain the $B_{\max }$ location. 


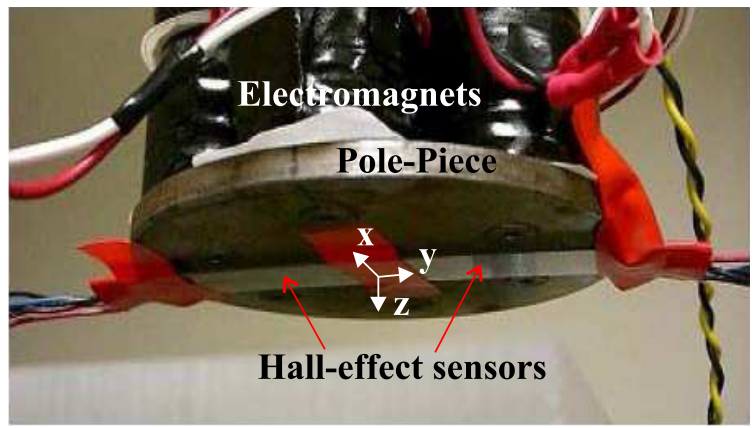

Fig. 5 The Hall-effect sensors are attached to a measurement stick and installed on the bottom of pole-piece ${ }^{(18)}$

\section{4. $B_{\max }$ Position Determination}

In Ref. (18), we have proposed a novel technique to determine the microrobot's position by measuring the magnetic flux produced by the MDU. The MDU aligns the magnetic field in such a way that only one $B_{\max }$ emerges in the horizontal plane below the pole-piece. The position of $B_{\max }$ can be controlled by tunning the electromagnets' produced magnetic fluxes [changing the electromagnets' coil current alters produced magnetic flux]. Thus, the $B_{\max }$ 's horizontal shifts are took place by the produced magnetic flux. If the relation is reversed, the $B_{\max }$ horizontal position can be estimated by measuring the magnetic flux. This reverse concept can be employed to determine the $B_{\max }$ location.

A combination of Hall-effect sensors has been installed on the MDU's structure to measure the magnetic flux. Since the generated magnetic flux of electromagnets penetrates to the MMS's workspace through the pole-piece, Hall-effect sensors are attached to the pole-piece to measure the produced magnetic flux. In Ref. (18), we have extensively investigated the optimal arrangement of Hall-effect sensors. In order to estimate the $B_{\max }$ position in one axis, it is found that at least two hall sensors should be installed on the specified axis on the pole-piece. The difference of magnetic flux measured by these two sensors can be mapped to the $B_{\max }$ position; Fig. 5 shows the two Hall-effect sensors in th y-direction attached to the pole-piece.

The MDU has been used to manipulate the microrobot along the y-axis; meanwhile the magnetic flux is measured by the Hall-effect sensors and is recorded. Figure 6 presents the measured flux differential versus the microrobot's position on the y-direction. This figure also shows the best fitted curves to experimental measurements as

$$
y_{\text {Bmax }}=0.044 v_{d}+0.0069
$$

where $v_{d}$ is the flux differential in voltage measured by the Hall-effect sensors (Two hall sensors' outputs have been amplified via operational amplifier). The performance of Hall-effectbased $B_{\max }$ location estimation method in y-axis has been presented in Fig. 7. As shown in this figure, the Hall-effect sensors measurements coupled with the mathematical Eq. 13 provides accurate position estimation when the microrobot is close to the center of the working envelope, an accuracy of $0.3 \mathrm{~mm}$ as the root-mean-square (RMS) of the position error.

\section{Experimental Validation And Force Calibration}

Section 3 discussed the environmental force model -equals to the magnetic force in the steady-state condition-, which is linearly the function of the distance of the microrobot from the $B_{\max }$ location, Eq. 12. Section 4 discussed the technique for estimating the $B_{\max }$ location in the y-direction, Eq. 13. Replacing the $B_{\max }$ location, Eq. 13 , in the force model relation, Eq. 12, results in the following derivation of the force

$$
f_{y}=f_{e}=C \Delta y=C\left(y_{r}-y_{B_{m} a x}\right)=C\left(y_{r}-0.044 v_{d}-0.0069\right)=C_{r} y_{r}+C_{v} v_{d}+C_{0}
$$




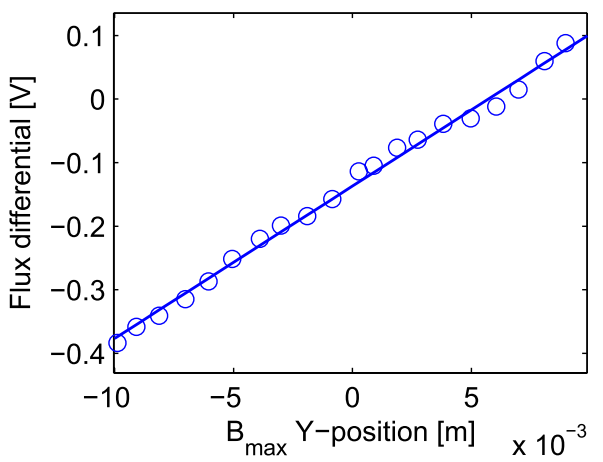

Fig. 6 The changes of $B_{\max }$ location in y-direction versus variation of magnetic flux differential, position-voltage relation

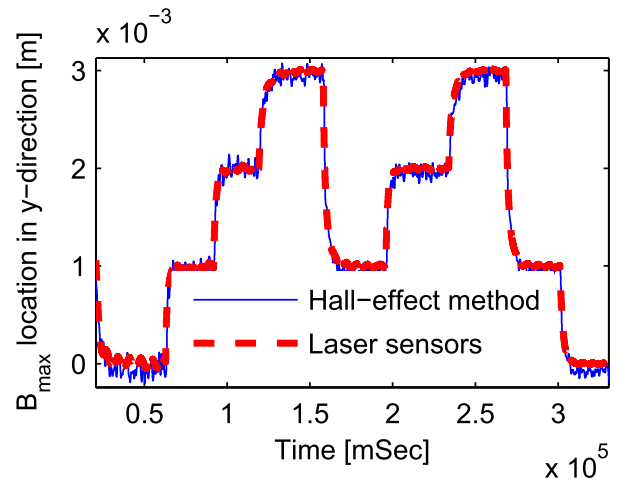

Fig. 7 The performance of using hall-effect sensors in estimating the microrobot's position

where $C_{r}, C_{v}$, and $C_{0}$ are constant coefficients that can be determined by a series of experimental measurements. Since Eq. 14 has three coefficients, these coefficients can be determined by defining at least three equations. Depending on the number of calibration points, the coefficients of the Eq. 14 can then be determined as

$$
\left[\begin{array}{c}
C_{r} \\
C_{v} \\
C_{0}
\end{array}\right]=\left[\begin{array}{ccc}
y_{r_{1}} & v_{d_{1}} & 1 \\
y_{r_{2}} & v_{d_{2}} & 1 \\
\vdots & \vdots & 1 \\
y_{r_{n-1}} & v_{d_{n-1}} & 1 \\
y_{r_{n}} & v_{d_{n}} & 1
\end{array}\right]^{\dagger}\left[\begin{array}{c}
f_{y_{1}} \\
f_{y_{2}} \\
\vdots \\
f_{y_{n-1}} \\
f_{y_{n}}
\end{array}\right]
$$

where the subscript $i$ denotes the number of the calibration point, and $\dagger$ is the generalized inverse or pseudo-inverse operation of a matrix. To obtain calibration points, a high precision force measurement setup has been designed based on the tip deflection of a cantilever, Fig. 8 shows this setup. The microrobot used for this study includes a cylindrical permanent magnet, the radius of $5 \mathrm{~mm}$ and the height of $10 \mathrm{~mm}$, as the head of the microrobot with a needle-base end-effector. The weight of this microrobot is $11 \mathrm{gr}$. The aluminum cantilever is $50 \times 5 \times 0.05$ $\mathrm{mm}^{3}$ and made by Aluminum alloy 1100. The laser sensor measures the tip deflection of the cantilever with the accuracy. The force applied to the tip of the cantilever can be obtained by

$$
P=\frac{3 E I \delta}{l^{3}}
$$

where $E, I, l$, and $\delta$ are modulus of elasticity, area moment of inertia, length, and elastic deflection, respectively. The accuracy analyses demonstrated that the cantilever-based force measurement method provides the error of less than $0.64 \mu \mathrm{N}$ in measuring the applied force to the cantilever's tip. However, this error can be reduced by increasing the accuracy of deflection measurement and physical properties of the cantilever. In the experimental measurement, 


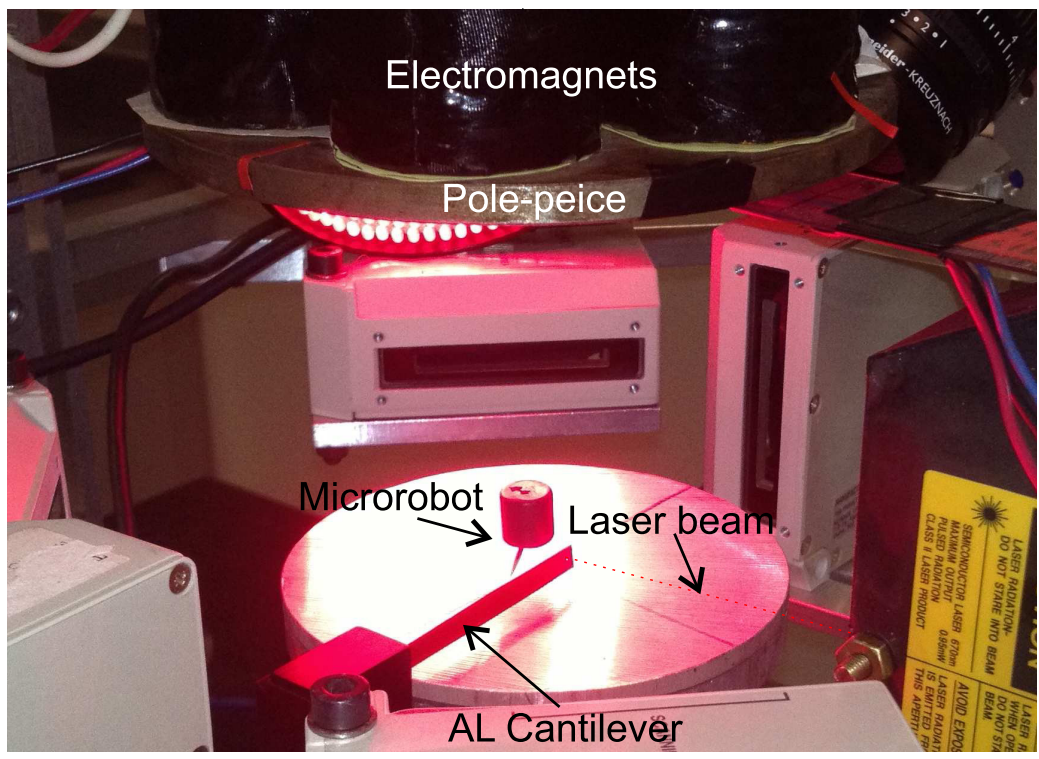

Fig. 8 Force measurement setup-using cantilever tip deflection

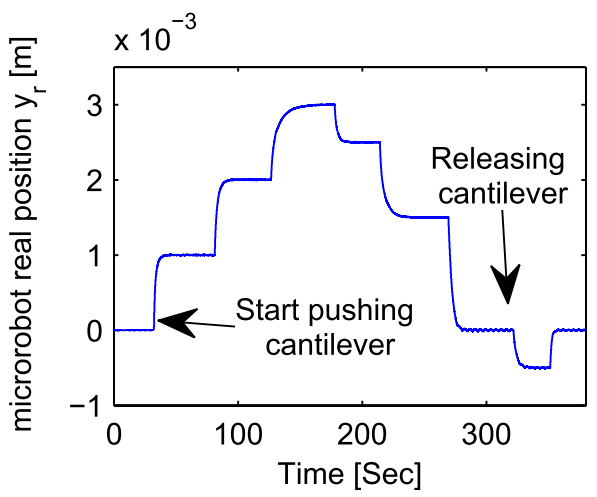

(a)

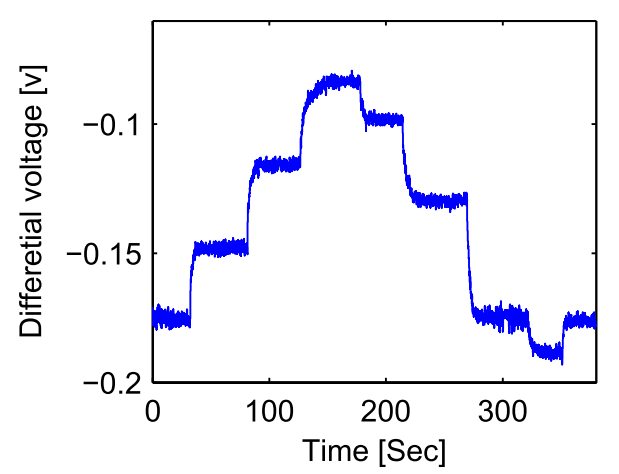

(b)

Fig. 9 The microrobot is commanded toward the cantilever's tip: a) real position of the microrobot b) flux differential in voltage

the cantilever is placed with zero distance from the microrobot, and the microrobot is then commanded toward the cantilever's tip in several steps and then moved backward to detach from the cantilever's tip. As shown in Fig. 9, the microrobot starts pushing moderately the cantilever's tip at the time of 32.3 second by increasing the magnetic flux differential [the flux differential changes in voltage $\left(v_{d}\right)$ versus the real position of the microrobot $\left(y_{r}\right)$ is presented in Fig. 9.b], and the deflection measurement records first displacement of the cantilever's tip at time of the 32.7 second. The microrobot is detached form the cantilever's tip at the time 321 second, since the microrobot is commanded to move to the position $-0.5 \mathrm{~mm}$. Although the releasing process has softly taken place, a small vibration occurs in the cantilever as seen in Fig. 10 which presents the deflection of the cantilever's tip and the measured force by Eq. 16.

To calculate the force by using Eq. 14, the flux differential in voltage and the real position of the microrobot are instantaneously recorded during the experimental measurements, previously demonstrated by Fig. 9.b. Using Eq. 15 and the obtained calibration points, the unknown coefficients of Eq. 14 can then be determined as $C_{r}=-5.47 \times 10^{-3}, C_{v}=2.42 \times 10^{-5}$, and $C_{0}=4.09 \times 10^{-6}$. The force applied to the cantilever's tip can be calculated by the Halleffect sensors method by replacing the derived coefficients in Eq. 14, shown in Fig. 11 the comparison of the two force measurement methods: measuring the deflection of the cantilever and the Hall-effect-based measurement. As shown in this figure, the Hall-effect-based method 


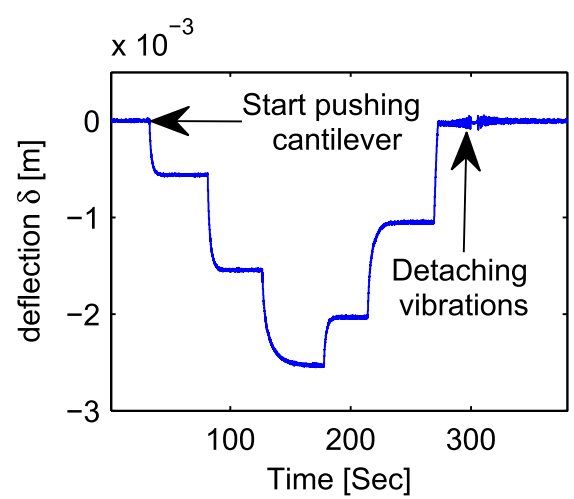

(a)

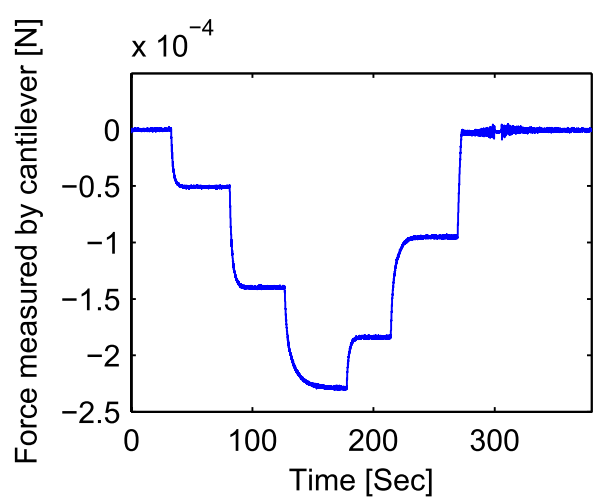

(b)

Fig. 10 The microrobot is commanded toward the cantilever's tip: a) cantilever's tip deflection b) force estimated based on the measured deflection

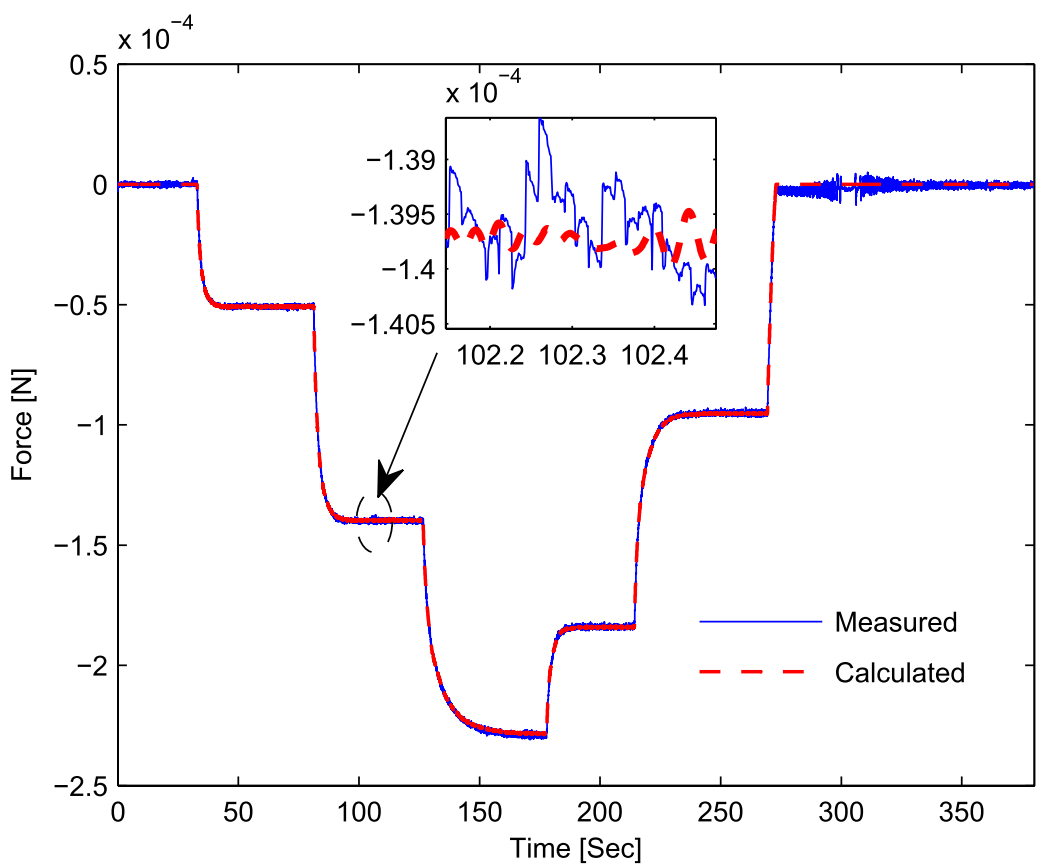

Fig. 11 The comparison of the two force measurement methods: measured by the deflection of the cantilever and calculated by the Hall-effect-based method

can accurately estimate the deflection force. To validate the performance of Hall-effect-based method corresponding to the cantilever-based technique, the root-mean-square (RMS) of the force error, calculated force obtained by Hall-effect-based method minus the measure force obtained by the cantilever-based technique, has been calculated as $0.63 \mu \mathrm{N}$. Thus, the noncontact force estimation technique, Hall-effect-based method, provides high accuracy relative to the measured force by the cantilever-based routine. Since the cantilever-based technique has the accuracy of $0.64 \mu \mathrm{N}$, the accuracy of Hall-effect-based method can be calculated as $1.27 \mu \mathrm{N}$.

The concept of Hall-effect-based force measurement, in Section 4, is based on the distance of real position of the microrobot and the estimated $B_{\text {max }}$ location, Fig. 12 demonstrates the change of $B_{\max }$ location versus the microrobot's real position, when the microrobot pushes the cantilever. As expected, by increasing the cantilever's deflection, the distance of the microrobot's position from the $B_{\max }$ location becomes larger. As shown, when the microrobot is in contact with cantilever, there is discrepancy between the microrobot's position and the estimated $B_{\max }$ location, and whenever the microrobot is detached from the cantilever, the microrobot's position and the estimated $B_{\max }$ are very closed to each other, Fig. 13 presents the 


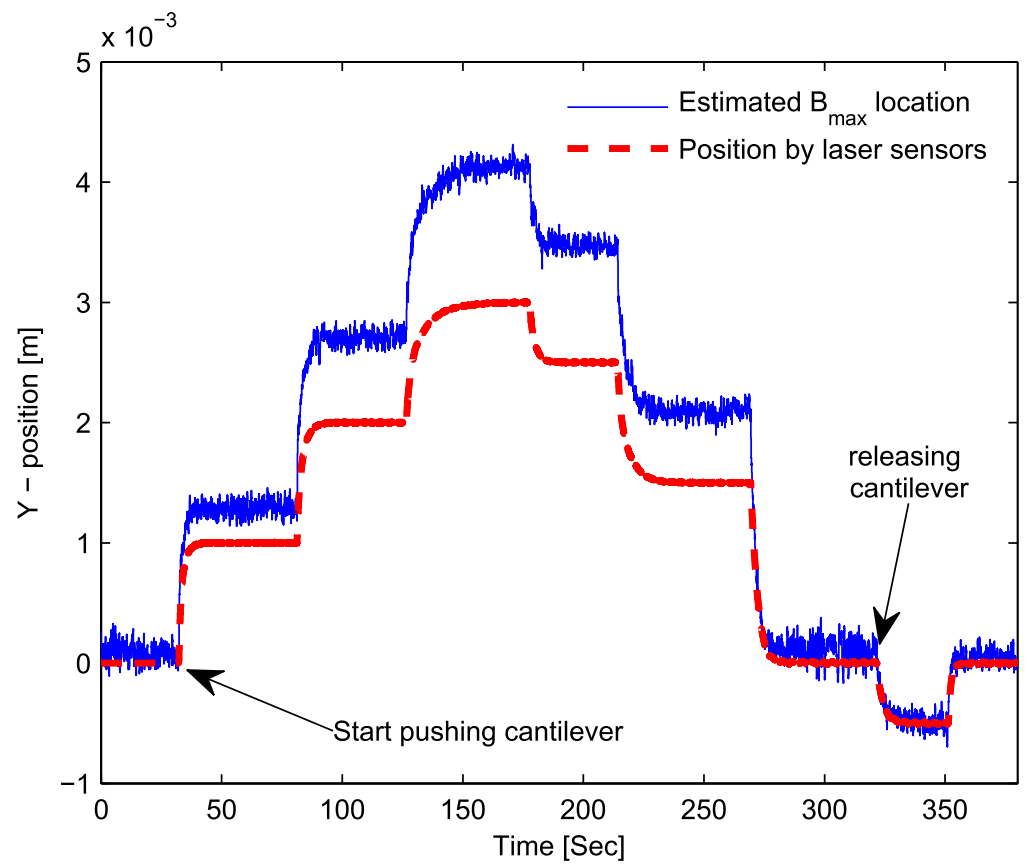

Fig. 12 The microrobot's position versus estimated $B_{\max }$ location- the cantilever is placed with zero distance distance from the microrobot

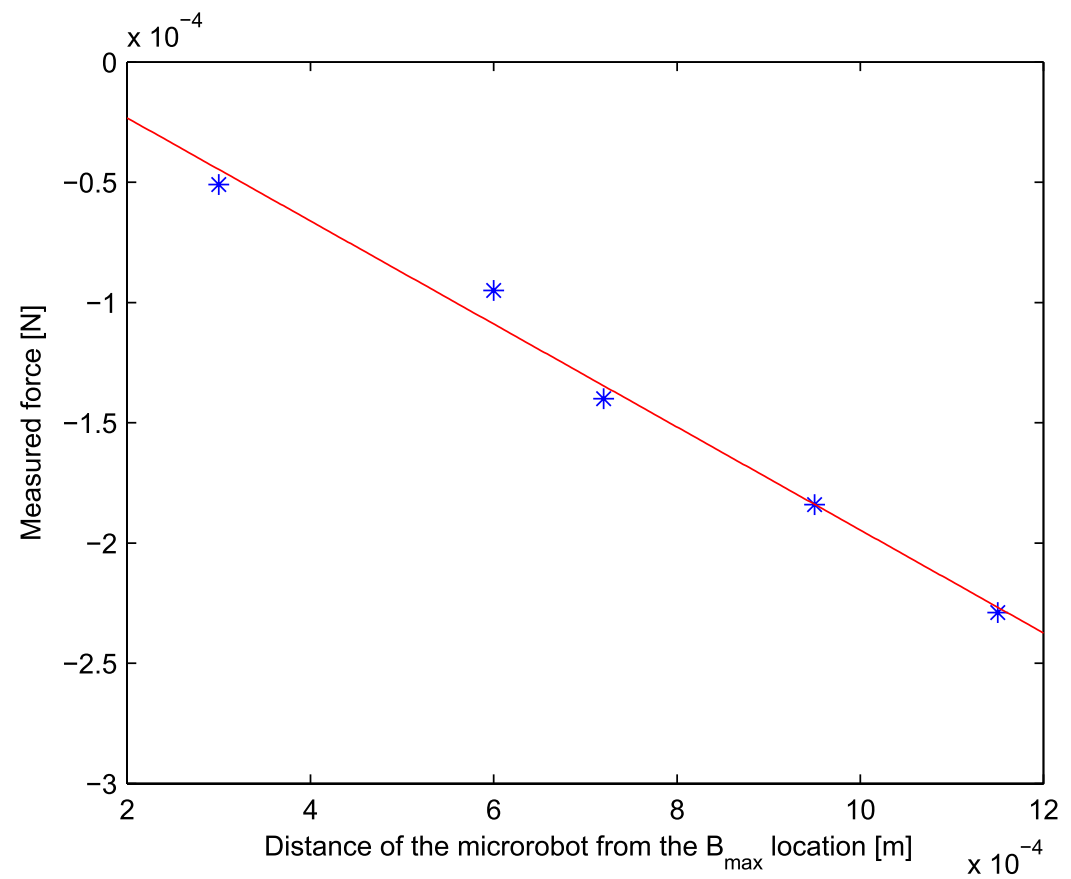

Fig. 13 Changes of force in respect to the distance of the microrobot from the $B_{\max }$ location

linear relation of applied force to the microrobot by measuring the distance of the microrobot from the estimated $B_{\max }$.

\section{6. conclusion}

In this study, a novel micro-domain force measurement methodology is proposed for a magnetic-haptic micromanipulation platform (MHMP). The MHMP consists of a micro magnetically levitated slave robot and a macro-master haptic robot. The MHMP enables a human operator to control the micro slave robot by manoeuvring the macro-master haptic robot. To allow the human operator the feeling of a micro-domain task, the micro-domain force estima- 
tion method is developed to measure the environmental force in a single-axis direction. This method uses magnetic flux measurement and the microrobot's position information to calculate the environmental force. No force sensor is attached to the microrobot to measure the force, that keeps the microrobot's size restriction and makes the microrobot inexpensive and disposable for biological applications.

\section{Acknowledgements}

The authors gratefully acknowledge the Canada Foundation for Innovation (CFI) and the Natural Science and Engineering Research Council of Canada (NSERC) for financial support.

\section{References}

( 1 ) H. Zhang, E. Burdet, A.N. Poo, and D.W. Hutmacher, Microassembly fabrication of tissue engineering scaffolds with customized design, Automation Science and Engineering, IEEE Transactions on, 5(3):446-456, 2008.

( 2 ) X. Liu, K. Kim, Y. Zhang, and Y. Sun, Nanonewton force sensing and control in microrobotic cell manipulation, The international journal of robotics research, 28(8):10651076, 2009.

( 3 ) R.A. MacLachlan, B.C. Becker, J. Cuevas Tabares, G.W. Podnar, L.A. Lobes, and C.N. Riviere, Micron: an actively stabilized handheld tool for microsurgery, Robotics, IEEE Transactions on, 28(1):195-212, 2012.

( 4 ) S. Hosseini, M. Mehrtash, and M.B. Khamesee, Design, fabrication and control of a magnetic capsule-robot for the human esophagus, Microsystem technologies, 17(5):1145-1152, 2011.

( 5 ) M. Mehrtash, N. Tsuda, and M.B. Khamesee, Bilateral macro-micro teleoperation using magnetic levitation, Mechatronics, IEEE/ASME Transactions on, 16(3):459-469, 2011.

( 6 ) H.J. Butt, B. Cappella, and M. Kappl, Force measurements with the atomic force microscope: Technique, interpretation and applications, Surface Science Reports, 59(1):1$152,2005$.

( 7 ) T.C. Duc, JF Creemer, and P.M. Sarro, Piezoresistive cantilever beam for force sensing in two dimensions, Sensors Journal, IEEE, 7(1):96-104, 2007.

( 8 ) Hui Xie and Stphane Rgnier, Three-dimensional automated micromanipulation using a nanotip gripper with multi-feedback, Journal of Micromechanics and Microengineering, 19(7):075009, 2009.

( 9 ) J.C. Doll, S.J. Park, and B.L. Pruitt. Design optimization of piezoresistive cantilevers for force sensing in air and water, Journal of applied physics, 106(6):064310-064310, 2009.

(10) S.J. Park, J.C. Doll, and B.L. Pruitt, Piezoresistive cantilever performancepart i: Analytical model for sensitivity, Microelectromechanical Systems, Journal of, 19(1):137-148, 2010.

(11) S.J. Park, J.C. Doll, A.J. Rastegar, and B.L. Pruitt, Piezoresistive cantilever performancepart ii: optimization, Microelectromechanical Systems, Journal of, 19(1):149-161, 2010.

(12) S.J. Park, B.C. Petzold, M.B. Goodman, and B.L. Pruitt, Piezoresistive cantilever forceclamp system, Review of Scientific Instruments, 82(4):043703-043703, 2011.

(13) T. Hoffmann, B. Eilebrecht, and S. Leonhardt, Respiratory monitoring system on the basis of capacitive textile force sensors, Sensors Journal, IEEE, 11(5):1112-1119, 2011.

(14) L. Ni and D.W.L. Wang, A gain-switching control scheme for position-error-based bilateral teleoperation: Contact stability analysis and controller design, The International Journal of Robotics Research, 23(3):255-274, 2004.

(15) M. Tavakoli, R.V. Patel, M. Moallem, and A. Aziminejad, Haptics for teleoperated surgical robotic systems, World Scientific, 2008.

(16) M.B. Khamesee, N. Kato, Y. Nomura, and T. Nakamura, Design and control of a micro- 
robotic system using magnetic levitation, Mechatronics, IEEE/ASME Transactions on, 7(1):1-14, 2002.

(17) M. Mehrtash and M.B. Khamesee, Design and implementation of lqg $\backslash$ ltr controller for a magnetic telemanipulation system-performance evaluation and energy saving, Microsystem technologies, 17(5):1135-1143, 2011.

(18) M. Mehrtash, M.B. Khamesee, N. Tsuda, and J.Y. Chang, Motion control of a magnetically levitated microrobot using magnetic flux measurement, Microsystem Technologies, 18:1417-1424, 2012. 